\title{
A needle in a needlestack: exploiting functional inhomogeneity for optimized nanowire lasing
}

Parkinson, Patrick, Alanis, Juan, Skalsky, Stefan, Zhang, Yunyan, Liu, Huiyun, et al.

Patrick Parkinson, Juan A. Alanis, Stefan Skalsky, Yunyan Zhang, Huiyun Liu, Mykhaylo Lysevych, Hark Hoe Tan, Chennupati Jagadish, "A needle in a needlestack: exploiting functional inhomogeneity for optimized nanowire lasing," Proc. SPIE 11291, Quantum Dots, Nanostructures, and Quantum Materials: Growth, Characterization, and Modeling XVII, 112910K (2 March 2020); doi: 10.1117/12.2558405

SPIE. Event: SPIE OPTO, 2020, San Francisco, California, United States 


\title{
A needle in a needlestack: exploiting functional inhomogeneity for optimized nanowire lasing
}

\author{
Patrick Parkinson ${ }^{\mathrm{a}}$, Juan A. Alanis ${ }^{\mathrm{a}}$, Stefan Skalsky ${ }^{\mathrm{a}}$, Yunyan Zhang ${ }^{\mathrm{b}}$, Huiyun Liu ${ }^{\mathrm{b}}$, Mykhaylo \\ Lysevych $^{\mathrm{c}}$, Hark Hoe Tanc ${ }^{\mathrm{c}}$, and Chennupati Jagadish ${ }^{\mathrm{c}}$ \\ aDepartment of Physics and Astronomy and the Photon Science Institute, University of \\ Manchester, Oxford Road, Manchester, United Kingdom \\ ${ }^{\mathrm{b}}$ Department of Electronic Engineering, University College London, London, United Kingdom \\ ${ }^{\mathrm{c}}$ Electronic Materials Engineering, Research School of Physics, Australian National University, \\ Canberra, Australia
}

\begin{abstract}
III-V semiconductor nanowires allow easy hetero-integration of optoelectronic components onto silicon due to efficient strain relaxation, well-understood design approaches and scalability. However continuous room temperature lasing has proven elusive. A key challenge is performing repeatable single-wire characterization - each wire can be different due to local growth conditions present during bottom-up growth. Here, we describe an approach using large-scale population studies which exploit inherent inhomogeneity to understand the complex interplay of geometric design, crystal structure, and material quality. By correlating nanowire length with threshold for hundreds of nanowire lasers, this technique reveals core-reabsorption as the critical limiting process in multiple-quantum-well nanowire lasers. By incorporating higher band-gap nanowire core, this effect is eliminated, providing reflectivity dominated behavior.
\end{abstract}

Keywords: Semiconductor Nanowires, Nanolasers, Statistical Optimization

\section{INTRODUCTION}

Growth of semiconductor nanowires using bottom-up epitaxy is now a well-developed technology. ${ }^{1}$ By using seeded, ${ }^{2}$ templated, ${ }^{3}$ or gas-phase synthesis ${ }^{4}$ growth, a variety of functional optoelectronic devices have been demonstrated. While devices which exploit the ensemble properties of nanowires are of great interest for coherent emission, ${ }^{5}$ photodetection ${ }^{6}$ and photovoltaics,${ }^{7}$ the potential for novel single element nanowire devices provides unprecedented possibilities for integration ${ }^{8}$ or entirely new devices. ${ }^{9}$ Since the first development of semiconductor nanowires materials, the possibility of using nanowires as both cavity and gain material in nanoscale coherent light sources - or nanolasers - has been recognized. ${ }^{10}$ This development has led to both deeper fundamental understanding ${ }^{11}$ and applications with relevance for high-density integration and chip-tochip communication. ${ }^{12,13}$ Despite this promise, designing and optimizing nanowires for lasing applications has proved to be a significant challenge, primarily due to the complex interplay of material quality, optoelectronic behavior (including surface recombination) and geometrical effects which combine to determine lasing performance. While the past decade has seen significant advances in growth for high crystallographic quality, ${ }^{14}$ surface passivation, ${ }^{15,16}$ radial quantum-well ${ }^{17,18}$ growth, axial ${ }^{19,20}$ or self-assembled quantum-dots ${ }^{21}$ integration, and understanding catalyst dynamics, ${ }^{22}$ room-temperature continuously operating III-V nanowire lasers have not yet been demonstrated. ${ }^{13,18,23}$

The primary figure of merit for pulsed, optical-pumped nanowire lasers is the lasing threshold defined as the pump fluence required to produce coherent lasing emission from the wires $\left(P_{\text {las }}\right)$. The relationship between $P_{\text {las }}$ and the material gain and cavity properties of a nanowire laser is highly nonlinear. Because nanowires typically have wavelength-scale diameters they act as waveguides; the end-facet cavity reflectivity in a nanowire laser is a complex function of the facet structure, nanowire diameter, the transverse lasing mode, and the

Further author information: (Send correspondence to P.P.)

P.P.: E-mail: patrick.parkinson@manchester.ac.uk

Quantum Dots, Nanostructures, and Quantum Materials: Growth, Characterization, and Modeling XVII, edited by Diana L. Huffaker, Holger Eisele, Proc. of SPIE Vol. 11291, 112910K

(c) 2020 SPIE $\cdot$ CCC code: $0277-786 X / 20 / \$ 21 \cdot$ doi: $10.1117 / 12.2558405$

Proc. of SPIE Vol. 11291 112910K-1 
refractive index of the medium. ${ }^{24}$ As such, it is common for a given growth of nanowire lasers to have a large wire-to-wire variation in emission ${ }^{25}$ or lasing performance. ${ }^{26}$ This spread can mask optimizations in underlying material properties or gain, which might otherwise be clearly observed. ${ }^{27}$

In this context, the prospects for systematic optimization of nanolasers may appear challenging. Here, we propose that, far from being a hindrance, the inherent spread in material and geometric properties arising from bottom-up growth can in fact be exploited to provide a statistically relevant understanding of lasing. To achieve this, we have developed an automated machine-vision enabled data acquisition approach to scale characterization to the $>10^{3}$ nanowire level, ${ }^{26}$ used computational approaches typically used in astrophysics to perform markerfree multi-modal characterization, ${ }^{28}$ and begun to use statistical methods from the life sciences ${ }^{29}$ to systematically optimize nanowire lasers in the presence of inhomogeneity. In this communication, we describe the experimental and statistical approaches used to characterize the dominant loss mechanism in silicon integrable nanolasers based on a multiple-quantum-well gain region. By studying the relationship between lasing and nanowire length for a large population of nanowires, we are able to identify core-reabsorption as a critical limiting process. Using this knowledge, nanowires without core-reabsorption were grown with a significantly reduced lasing threshold of $10 \mu \mathrm{Jcm}^{-2}$ pulse $^{-1}$.

\section{EXPERIMENTAL DETAILS}

Semiconductor nanowires were grown either homoepitaxially using the vapor-liquid-solid mechanism and MOVPE (Type 1: full recipe and growth details in 30), or heteroepitaxially onto Silicon using a foreign-catalyst free approach in MBE (Type 2: full recipe and growth details in 18). After growth, both nanowire types were transferred to quartz discs by gently rubbing the growth substrate onto the receiver or fast ultrasonication into solvent and re-deposition from solution. These approaches are known to produce high yield lasing. ${ }^{29}$ Briefly, type 1 wires have an $80 \mathrm{~nm}$ GaAs core, eight $\mathrm{Al}_{x} \mathrm{Ga}_{(1-x)} \mathrm{As} / \mathrm{GaAs}$ quantum wells (with $x=0.42$ and a nominal thickness of $3.5 \pm 1.5 \mathrm{~nm}$ ), and a final $5 \mathrm{~nm}$ GaAs capping layer. ${ }^{26,30}$ These wires had typical final dimensions of $3.4 \pm 0.4 \mu \mathrm{m}$ length and $460 \pm 10 \mathrm{~nm}$ diameter. Type 2 nanowires had $\sim 50 \mathrm{~nm} \mathrm{GaAs} \mathrm{P}_{(1-x)}$ cores with $x=0.47$, three GaAs/ $\operatorname{GaAs}_{x} \mathrm{P}_{(1-x)}$ quantum wells (with $x=0.62$ in the barriers, and a nominal well thickness varying from 3.5-10 nm), an AlGaAsP passivation layer and a final GaAsP capping layer. The wires were $\sim 10 \mu \mathrm{m}$ in length and approximately $400 \mathrm{~nm}$ in diameter. ${ }^{18}$

\subsection{EXPERIMENTAL DETAILS}

The substrates with nanowires were transported to a home-built optical microscope equipped with machine vision camera and motorized sample stage, with routing for laser excitation and quasi-confocal fibre-optic detection as shown in Figure 1. A sequential approach was take to collect the full population data sets:

Imaging: Bright-field or dark-field optical micrograph images were taken, and for each image nanowire features were identified and recorded using a machine vision approach derived from 31 . The image of each wire, and the absolute position was recorded for $\sim 10 \mathrm{k}$ nanowires. Typical raw images are shown below the inset of Figure 1.

Selection 1: The set of identified objects were reduced by cutting non-wire objects; this is achieved by placing physical limits on lengths, widths and areas of the imaged ensemble.

Low-power optical spectroscopy: A lower power HeNe laser was used to excite the nanowires sequentially, and the photoluminescence was collected using the fibre-coupled optical spectrometer.

Selection 2: Non-emitting objects are excluded to eliminate dust or other foreign contaminants. A subset of 100-1000 wires is then randomly selected for in-depth study. 


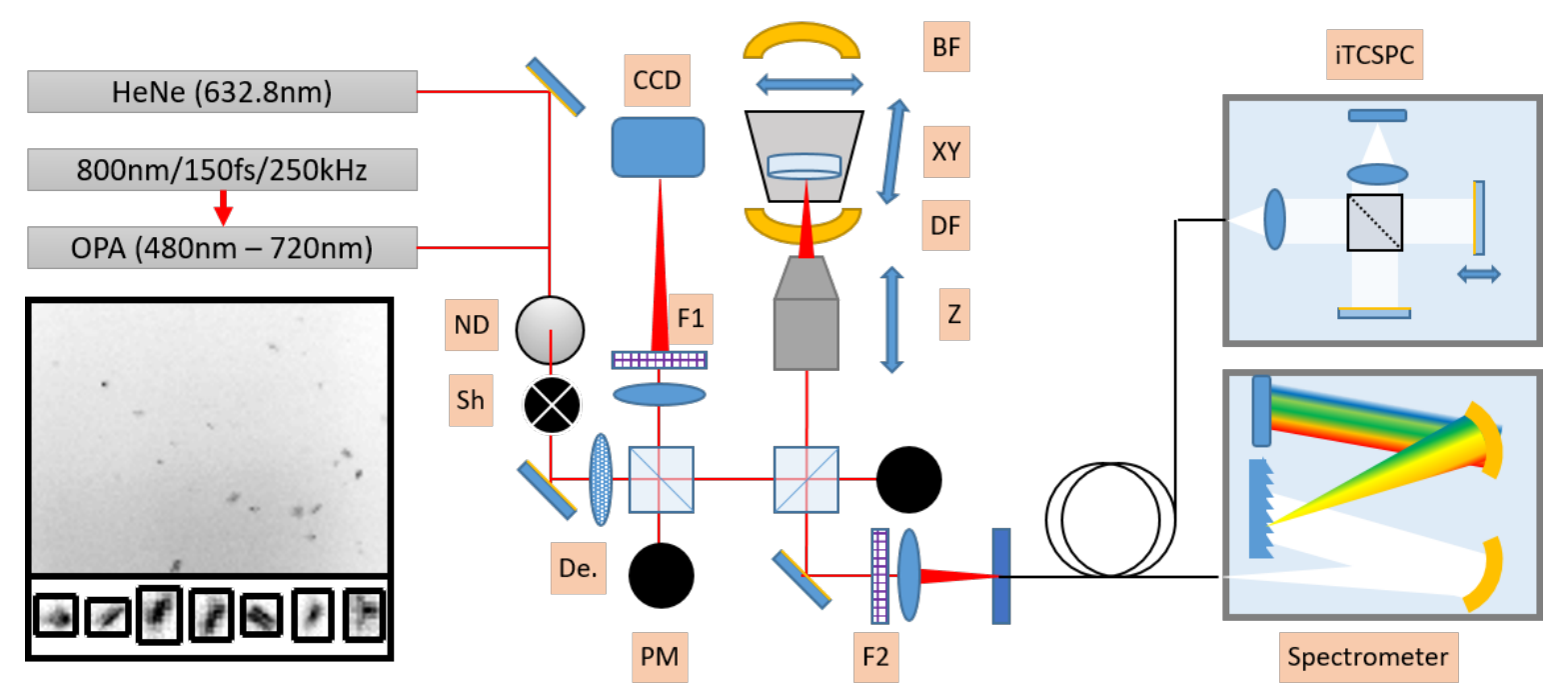

Figure 1. A schematic showing the experimental arrangement for automated micro-spectroscopy. Two laser sources are used - HeNe for continuous, low power spectroscopy and a femtosecond pulsed OPA for lasing study. ND: automated neutral density filter, Sh: Shutter, De.: (optional) defocusing lens, PM: power meter, F1: long-pass filter, CCD: camera, Z: motorized z-focus, DF: Dark-field lighting, XY: motorized translation stage, BF: Bright-field lighting, F2: long-pass filter 2, Spectrometer: Fibre coupled, cooled CCD, iTCSPC: interferometric time-correlated single photon counting. (Inset, bottom left): A typical bright-field microscopy image.

Optimization: The alignment for each nanowire is programatically optimized by raster scanning the excitation spot over the nanowire area and monitoring the emission intensity. A fluorescence image is taken for each wire and recorded.

Time-resolved emission: The excitation source is changed to a $620 \mathrm{~nm}, 150 \mathrm{fs}, 250 \mathrm{kHz}$ laser delivering up to $2000 \mu \mathrm{Jcm}^{-2}$ pulse ${ }^{-1}$ at full power. At a low fluence, the emission from each wire can be monitored using time-correlated single photon counting (TCSPC) coupled to an interferometer for Hanbury-Brown-Twiss type measurements for bunching or anti-bunching studies.

Power-dependent photoluminescence: By stepping the laser power and recording the photoluminescence spectra, power-in vs power-out data is recorded. The experiment is stopped if the laser emission decreases, or where lasing is confirmed over sequentional measurements. The onset of lasing is programatically identified by photoluminescence clamping, an increase in the gradient of the light-in vs light-out curve and the emergence of a narrow (sub-5nm) emission line.

Lasing image: When the lasing threshold is identified, a far-field image of the nanowire laser is taken. This can also be used to confirm coherent emission from the wire. Once the data is collected, the sample can be removed and additional off-line characterization approaches can be performed using a marker-free registration. ${ }^{28}$

\subsection{DATA PROCESSING}

The collected data for each wire is used to generate population-wide statistics. Figure 2 shows a typical data set for a single Type 1 nanowire laser; using this this, additional processing provides nanowire length, lasing wavelength, lasing threshold, and laser mode spacing. Further modeling can provide quantum well widths ${ }^{27}$ or composition, ${ }^{32}$ doping level ${ }^{33,34}$ or modal refractive index. ${ }^{28}$ At room-temperature, the low-fluence photoluminescence can be modeled as a function of emission energy $E$ :

$$
I_{3 \mathrm{D}}(E)=A_{3 \mathrm{D}}\left(G(E, \sigma) \circledast\left(\sqrt{\left(E-E_{0}\right)} B\left(E, E_{0}, T\right)\right)\right),
$$



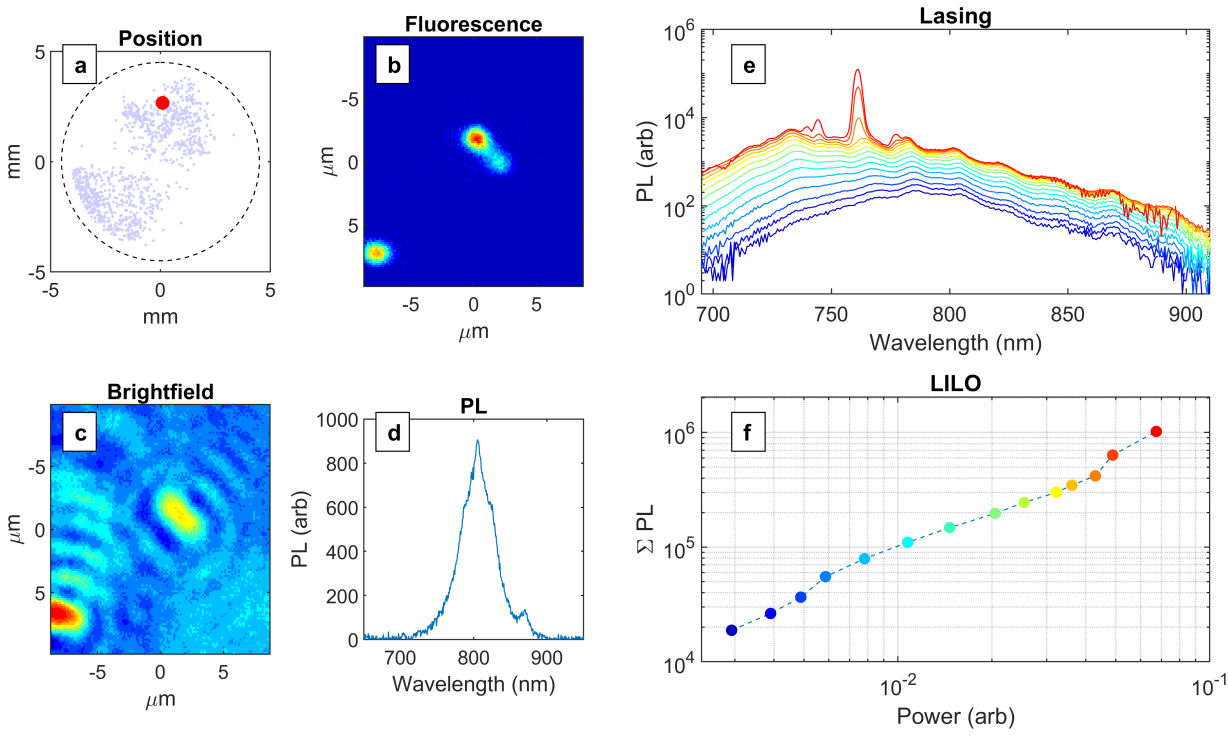

Figure 2. A typical data set acquired for each nanowire in an ensemble for Type 1 wires. The (a) position (example wire in red, other wires in gray), (b) bright-field and (c) photoluminescence images can be interpreted to provide geometric information. (d) Modeling the photoluminescence spectra provides information on quantum well parameters. (e) The lasing spectra provides the modal spacing, while (f) the light-in vs light-out (LILO) plot gives the lasing threshold, where the marker colors indicate the related spectrum in (e) and the line is a guide to the eye.

for a 3D density of states, or

$$
I_{2 \mathrm{D}}(E)=A_{2 \mathrm{D}}\left(G(E, \sigma) \circledast\left(B\left(E, E_{0}, T\right)\right)\right),
$$

for a $2 \mathrm{D}$ density of states, where $G(E, \sigma)=\exp \left(E^{2} / \sigma^{2}\right)$ represents Gaussian disorder, $B\left(E, E_{0}, T\right)=(E>$ $\left.E_{0}\right) \exp -\left(E-E_{0}\right) / k T$ is a Boltzmann distribution, $E_{0}$ is the material band-gap, $T$ is the electronic temperature and $\circledast$ indicates a circular convolution. A Gaussian disorder term is used here to capture the combined effects of spectrometer resolution and local inhomogeneity, and is chosen for simplicity of application. Using this, the material band-gap can be accurately measured; by comparing this with a reference, parameters including material doping can be calculated where relevant. ${ }^{33,35}$

For each nanowire, the measured and calculated parameters are recorded. While simple histograms of these parameters across the population are of use for identifying ensemble trends and best-in-class performance, higher order correlations can provide otherwise hidden relationships between controllable (for instance nanowire length and diameter) and dependent (for instance lasing threshold) measurements. The loss mechanism in a laser system can be associated with distributed loss $\alpha_{\text {dist }}$ (which scales with nanowire length) and end-facet related loss, such that the threshold gain per unit length is approximated by:

$$
g_{\text {thresh }}=\alpha_{\text {dist }}-\frac{\ln R_{1} R_{2}}{2 L},
$$

where $R_{1,2}$ are the end-facet reflectivities and $L$ is the cavity length. Here, we use the strength of correlation between inverse nanolaser length and nanolaser threshold to quantify the relative contribution of distributed and end-facet losses. The threshold gain is related to the threshold excitation fluence by $\nu P_{\text {las }}=g_{\text {thresh }}$ where a factor $\nu$ is expected to vary between nanowires accounting for absorption (due to geometry). The end facet reflectivities will also have a distribution which is expected to dominate for low $\alpha_{\text {dist }}$ nanowires. Nonetheless, given sufficient data we can apply linear statistical correlation to identify trends and their significance.

\section{RESULTS AND DISCUSSION}

Equation 3 can be rearranged into a form

$$
P_{\text {las }}=A-B L^{-1}
$$



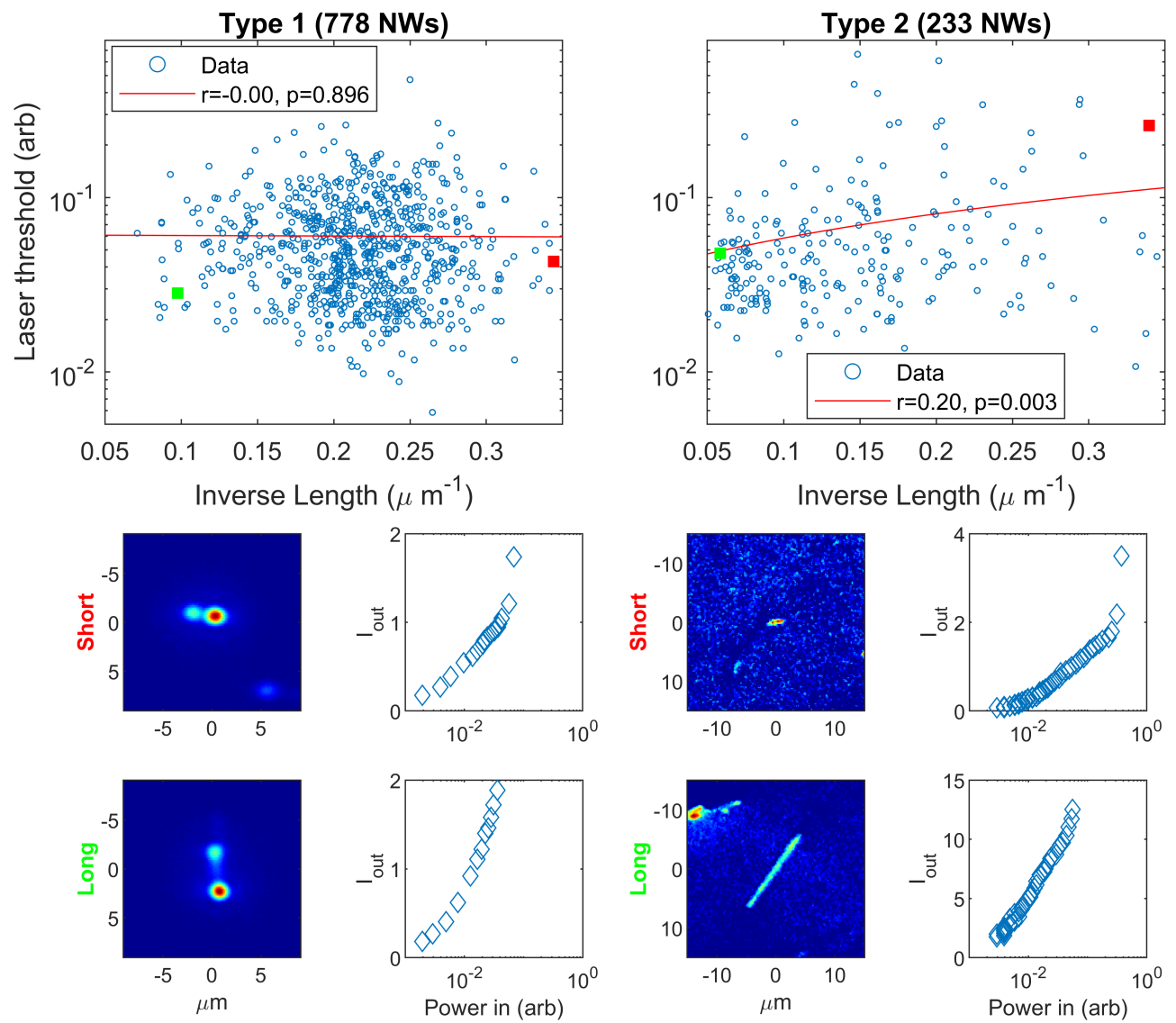

Figure 3. (Top) Scatter plots of inverse nanowire length against laser threshold for (left) 778 Type 1 nanowire lasers and (right) 233 Type 2 nanowire lasers. Each point represents a single laser. The red line is a linear best fit to the data, with the linear correlation coefficient $r$ and statistical significance $p$ given in the legend. The green and red squares indicate the wires corresponding to the Short and Long wires shown below. (Bottom) Images and light-in vs light-out curves for example short and long nanowire lasers from each type. Note the different length scale used for Type 1 and Type 2 wires.

where $A$ and $B$ are arbitrary parameters with unknown distributions. Figure 3 shows a scatter plot of $P_{\text {las }}$ as a function of inverse nanowire length $\left(L^{-1}\right)$ for the 778 Type 1 nanowires and 233 Type 2 nanowires which were identified as lasing. A linear correlation analysis on these data points shows that these two parameters appear uncorrelated for the Type 1 wires $(\rho \approx 0$ and $p \gg 0.01)$, but moderately correlated $(\rho=0.20$ at the $p<0.01$ significance level) for the Type 2 nanowires. This correlation analysis indicates that distributed losses dominate for Type 1 wires - no correlation is observed with length, meaning reflectivity does not play a significant role in determining threshold gain. On the other hand, end-facet reflectivity does play a role for Type 2 nanowire lasers - the correlation indicates that longer wires have lower threshold, as expected for end-facet dominated loss mechanisms. As a demonstration, four images and light-in vs light-out curves are shown for example short and long nanowire lasers for each of type 1 and Type 2 wires. Intriguingly, it can be seen for the Type 2 wires that the onset of lasing is sharp for the shorter wire, and less sharp for the longer wire. The onset of lasing is determined by the coupling of the spontaneous emission into the lasing mode $\beta$, with high $\beta$ operation typically associated with highly efficient lasing and a flat light-out vs light-in plot. ${ }^{36}$ Understanding and measuring $\beta$ factor is of critical importance to achieve continuous lasing, ${ }^{37}$ and further analysis of these results provides a fruitful opportunity for further study.

In both nanowire types, it is expected that the TE01 transverse mode is preferred. ${ }^{18,30}$ This is because 

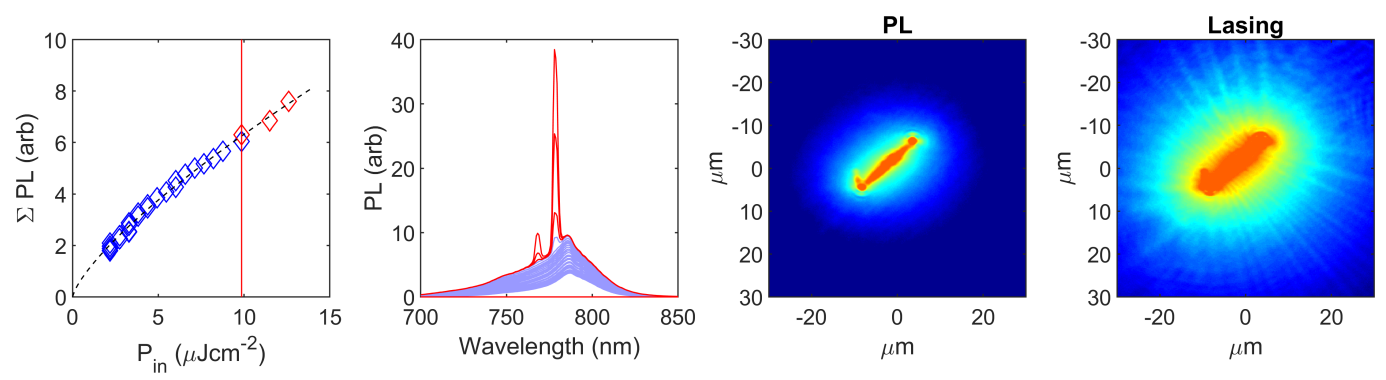

Figure 4. Selected low threshold nanowire from Type 2 study. From left to right: Light-out vs light-in curve; spontaneous (blue markers) and stimulated regions (red markers), with sub-threshold fit of the form $P L \propto P_{i n}^{\alpha}$ and $\alpha \sim-0.75$ with lasing threshold identified from the onset of lasing emission (vertical line). Spectral series, showing multimodal emission profile. Fluorescence image below and above the lasing threshold, showing coherent interference in the far-field.

this donut-type mode provides a high spatial overlap with the gain region provided by the radial quantum wells while remaining well confined by the dielectric properties of the nanowire. This mode has a radial intensity distribution of the form $I(\rho) \propto \rho \exp (-\rho)$ where $\rho=2 r^{2} w^{-2}, r$ is radial co-ordinate and $w$ is beam radius, which leads to a small but non-zero intensity as $r \rightarrow 0$. In this region, the lasing mode overlaps and interacts with the nanowire core, where re-absorption can occur if the core band-gap energy lies below the photon energy. This is the case for Type 1 nanowires, leading to a dominant photon loss mechanism that scales with the nanowire length. In the Type 2 nanowires, the use of a higher-band-gap core material mitigates this loss mechanism, leading to losses being less influenced by distribution reabsorption and more strongly influenced by the end facet reflectivity. While many factors play a role in determining the champion low-threshold emission, identification and hence elimination of the dominant source of loss is a powerful tool to close the loop between growth and characterization.

The large-scale statistical approach further allows the identification of best-in-class nanowire lasers. Results from one such nanolaser from the Type 2 growth is shown in Figure 4. Under the excitation conditions used, this wire exhibits a lasing threshold of around $10 \mu \mathrm{Jcm}^{-2}$ pulse $^{-1}$, which compares favorably with the best room-temperature near-infrared III-V nanowire lasers demonstrated.

\section{CONCLUSION}

By making use of the large populations present in semiconductor nanowire growth, population-based approaches can provide insight into dynamics and loss mechanisms that would otherwise be obscured by wire-to-wire variation. Here, we use a simple correlation of lasing threshold with nanowire length to infer the dominant source of photon loss in two nanowire types with the same architecture. For both GaAs-quantum well nanowire lasers, the lack of correlation between inverse length and threshold for GaAs-cored wires and positive correlation for GaAsP-cored wires is used to confirm that the primary loss mechanism is core-re-absorption. By changing the recipe to eliminate this re-absorption, lower-threshold lasing is demonstrated. This methodology is expected to provide a fertile area for further research; higher order correlations, more advanced statistical approaches, and Bayesian approaches can be used to experimentally determine fundamental laser properties in the presence of high wire-to-wire disorder. More generally, the large-scale spectroscopic approach is widely applicable for functional nanotechnology.

\section{ACKNOWLEDGMENTS}

We thank the Australian National Fabrication Facility (ANFF) ACT node for access to the epitaxial facility used in this work. JAA acknowledges a CONACyT-SENER funded scholarship. ML, HHT and CJ acknowledge financial support from the Australian Research Council. YZ and HL acknowledge the support of EPSRC grants EP/P000886/1, EP/P006973/1 and the EPSRC National Epitaxy Facility. 


\section{REFERENCES}

[1] N. P. Dasgupta, J. Sun, C. Liu, S. Brittman, S. C. Andrews, J. Lim, H. Gao, R. Yan, and P. Yang, "25th anniversary article: Semiconductor nanowires - Synthesis, characterization, and applications," Advanced Materials 26, pp. 2137-2183, 42014.

[2] H. J. Joyce, Q. Gao, H. H. Tan, C. Jagadish, Y. Kim, X. Zhang, Y. Guo, and J. Zou, "Twin-free uniform epitaxial GaAs nanowires grown by a two-temperature process," Nano Letters 7(4), pp. 921-926, 2007.

[3] H. Schmid, M. Borg, K. Moselund, L. Gignac, C. M. Breslin, J. Bruley, D. Cutaia, and H. Riel, "Templateassisted selective epitaxy of III-V nanoscale devices for co-planar heterogeneous integration with Si," Applied Physics Letters 106, p. 233101, 62015.

[4] M. Heurlin, M. H. Magnusson, D. Lindgren, M. Ek, L. R. Wallenberg, K. Deppert, and L. Samuelson, "Continuous gas-phase synthesis of nanowires with tunable properties," Nature 492, pp. 90-94, 122012.

[5] H. Kim, W.-J. Lee, A. C. Farrell, J. S. D. Morales, P. Senanayake, S. V. Prikhodko, T. J. Ochalski, and D. L. Huffaker, "Monolithic InGaAs Nanowire Array Lasers on Silicon-on-Insulator Operating at Room Temperature," Nano Letters 17, pp. 3465-3470, 2017.

[6] S. J. Gibson, B. van Kasteren, B. Tekcan, Y. Cui, D. van Dam, J. E. M. Haverkort, E. P. A. M. Bakkers, and M. E. Reimer, "Tapered InP nanowire arrays for efficient broadband high-speed single-photon detection," Nature Nanotechnology 14, pp. 473-479, 52019.

[7] N. Anttu, "Shockley-queisser detailed balance efficiency limit for nanowire solar cells," ACS Photonics 2, pp. 446-453, 12015.

[8] B. Tian, X. Zheng, T. J. Kempa, Y. Fang, N. Yu, G. Yu, J. Huang, and C. M. Lieber, "Coaxial silicon nanowires as solar cells and nanoelectronic power sources," Nature 449, pp. 885-889, 102007.

[9] K. Peng, P. Parkinson, J. L. Boland, Q. Gao, Y. C. Wenas, C. L. Davies, Z. Li, L. Fu, M. B. Johnston, H. H. Tan, and C. Jagadish, "Broadband Phase-Sensitive Single InP Nanowire Photoconductive Terahertz Detectors," Nano Letters 16, pp. 4925-4931, 82016.

[10] M. H. Huang, S. Mao, H. Feick, H. Yan, Y. Wu, H. Kind, E. Weber, R. Russo, and P. Yang, "RoomTemperature Ultraviolet Nanowire Nanolasers," Science 292(5523), pp. 1897-1899, 2001.

[11] R. Röder, T. P. H. Sidiropoulos, C. Tessarek, S. Christiansen, R. F. Oulton, and C. Ronning, "Ultrafast Dynamics of Lasing Semiconductor Nanowires," Nano Letters 15, pp. 4637-4643, 72015.

[12] B. Mayer, L. Janker, B. Loitsch, J. Treu, T. Kostenbader, S. Lichtmannecker, T. Reichert, S. Morkötter, M. Kaniber, G. Abstreiter, C. Gies, G. Koblmüller, and J. J. Finley, "Monolithically Integrated High- $\beta$ Nanowire Lasers on Silicon," Nano Letters 16, pp. 152-156, 12016.

[13] G. Koblmüller, B. Mayer, T. Stettner, G. Abstreiter, and J. J. Finley, "GaAs-AlGaAs core-shell nanowire lasers on silicon: invited review," Semiconductor Science and Technology 32, p. 053001, 52017.

[14] H. J. Joyce, Q. Gao, H. H. Tan, C. Jagadish, Y. Kim, M. A. Fickenscher, S. Perera, T. B. Hoang, L. M. Smith, H. E. Jackson, J. M. Yarrison-Rice, X. Zhang, and J. Zou, "High purity GaAs nanowires free of planar defects: Growth and characterization," Advanced Functional Materials 18(23), pp. 3794-3800, 2008.

[15] P. Parkinson, H. J. Joyce, Q. Gao, H. H. Tan, X. Zhang, J. Zou, C. Jagadish, L. M. Herz, and M. B. Johnston, "Carrier lifetime and mobility enhancement in nearly defect-free core-shell nanowires measured using time-resolved terahertz spectroscopy," Nano Letters 9(9), pp. 3349-3353, 2009.

[16] N. Jiang, Q. Gao, P. Parkinson, J. Wong-Leung, S. Mokkapati, S. Breuer, H. H. Tan, C. L. Zheng, J. Etheridge, and C. Jagadish, "Enhanced minority carrier lifetimes in GaAs/AlGaAs core-shell nanowires through shell growth optimization," Nano Letters 13, pp. 5135-5140, 112013.

[17] T. Shi, H. E. Jackson, L. M. Smith, N. Jiang, Q. Gao, H. H. Tan, C. Jagadish, C. Zheng, and J. Etheridge, "Emergence of localized states in narrow GaAs/AlGaAs nanowire quantum well tubes," Nano Letters 15, pp. 1876-1882, 32015.

[18] Y. Zhang, G. Davis, H. A. Fonseka, A. Velichko, A. Gustafsson, T. Godde, D. Saxena, M. Aagesen, P. W. Parkinson, J. A. Gott, S. Huo, A. M. Sanchez, D. J. Mowbray, and H. Liu, "Highly Strained III-V-V Coaxial Nanowire Quantum Wells with Strong Carrier Confinement," ACS Nano 13, pp. 5931-5938, 52019.

[19] J. Tatebayashi, S. Kako, J. Ho, Y. Ota, S. Iwamoto, and Y. Arakawa, "Room-temperature lasing in a single nanowire with quantum dots," Nature Photonics 9(8), pp. 501-505, 2015. 
[20] M. T. Borgström, V. Zwiller, E. Müller, and A. Imamoglu, "Optically bright quantum dots in single nanowires," Nano Letters 5(7), pp. 1439-1443, 2005.

[21] M. Heiss, Y. Fontana, A. Gustafsson, G. Wüst, C. Magen, D. D. O'Regan, J. W. Luo, B. Ketterer, S. ConesaBoj, A. V. Kuhlmann, J. Houel, E. Russo-Averchi, J. R. Morante, M. Cantoni, N. Marzari, J. Arbiol, A. Zunger, R. J. Warburton, and A. Fontcuberta I Morral, "Self-assembled quantum dots in a nanowire system for quantum photonics," Nature Materials 12, pp. 439-444, 52013.

[22] H. Gao, W. Sun, Q. Sun, H. H. Tan, C. Jagadish, and J. Zou, "Compositional Varied Core-Shell InGaP Nanowires Grown by Metal-Organic Chemical Vapor Deposition," Nano Letters 19, pp. 3782-3788, 52019.

[23] R. Röder and C. Ronning, "Review on the dynamics of semiconductor nanowire lasers," Semiconductor Science and Technology 33, p. 033001, 12018.

[24] A. V. Maslov and C. Z. Ning, "Reflection of guided modes in a semiconductor nanowire laser," Applied Physics Letters 83, pp. 1237-1239, 82003.

[25] S. Sergent, B. Damilano, S. Vézian, and S. Chenot, "Subliming GaN into ordered nanowire arrays for ultraviolet and visible nanophotonics," ACS Photonics 6, pp. 3321-3330, 122019.

[26] J. A. Alanis, D. Saxena, S. Mokkapati, N. Jiang, K. Peng, X. Tang, L. Fu, H. H. Tan, C. Jagadish, and P. Parkinson, "Large-Scale Statistics for Threshold Optimization of Optically Pumped Nanowire Lasers," Nano Letters 17, pp. 4860-4865, 82017.

[27] C. L. Davies, P. Parkinson, N. Jiang, J. L. Boland, S. Conesa-Boj, H. H. Tan, C. Jagadish, L. M. Herz, and M. B. Johnston, "Low Ensemble Disorder in Quantum Well Tube Nanowires," Nanoscale 7(48), pp. 2053120538, 2015.

[28] P. Parkinson, J. A. Alanis, K. Peng, D. Saxena, S. Mokkapati, N. Jiang, L. Fu, H. H. Tan, and C. Jagadish, "Modal refractive index measurement in nanowire lasers - a correlative approach," Nano Futures $\mathbf{2}$, p. 035004, 72018.

[29] J. A. Alanis, Q. Chen, M. Lysevych, T. Burgess, L. Li, Z. Liu, H. H. Tan, C. Jagadish, and P. Parkinson, "Threshold reduction and yield improvement of semiconductor nanowire lasers: Via processing-related endfacet optimization," Nanoscale Advances 1(11), pp. 4393-4397, 2019.

[30] D. Saxena, N. Jiang, X. Yuan, S. Mokkapati, Y. Guo, H. H. Tan, and C. Jagadish, "Design and RoomTemperature Operation of GaAs/AlGaAs Multiple Quantum Well Nanowire Lasers," Nano Letters 16, pp. 5080-5086, 82016.

[31] E. Bertin and S. Arnouts, "SExtractor: Software for source extraction," Astronomy and Astrophysics Supplement Series 117, pp. 393-404, 61996.

[32] T. E. Schlesinger and T. Kuech, "Determination of the interdiffusion of $\mathrm{Al}$ and $\mathrm{Ga}$ in undoped (Al,Ga)As/GaAs quantum wells," Applied Physics Letters 49, pp. 519-521, 91986.

[33] J. A. Alanis, M. Lysevych, T. Burgess, D. Saxena, S. Mokkapati, S. Skalsky, X. Tang, P. Mitchell, A. S. Walton, H. H. Tan, C. Jagadish, and P. Parkinson, "Optical Study of p-Doping in GaAs Nanowires for Low-Threshold and High-Yield Lasing," Nano Letters 19, pp. 362-368, 12019.

[34] N. H. Ky, L. Pavesi, D. Araújo, J. D. Ganière, and F. K. Reinhart, "A model for the Zn diffusion in GaAs by a photoluminescence study," Journal of Applied Physics 69, pp. 7585-7593, 61991.

[35] G. Borghs, K. Bhattacharyya, K. Deneffe, P. Van Mieghem, and R. Mertens, "Band-gap narrowing in highly doped n- and p-type GaAs studied by photoluminescence spectroscopy," Journal of Applied Physics 66, pp. 4381-4386, 111989.

[36] M. P. van Exter, G. Nienhuis, and J. P. Woerdman, "Two simple expressions for the spontaneous emission factor $\beta, "$ Physical Review A - Atomic, Molecular, and Optical Physics 54(4), pp. 3553-3558, 1996.

[37] B. Mayer, L. Janker, B. Loitsch, J. Treu, T. Kostenbader, S. Lichtmannecker, T. Reichert, S. Morkötter, M. Kaniber, G. Abstreiter, C. Gies, G. Koblmüller, and J. J. Finley, "Monolithically Integrated High- $\beta$ Nanowire Lasers on Silicon," Nano Letters 16, pp. 152-156, 12016. 